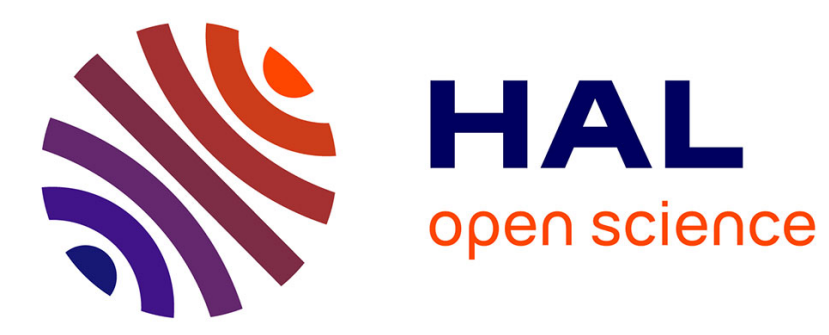

\title{
Numerical Simulation Of Granular Media Under Horizontal Vibrations.
}

Sébastien Nadler, Olivier Bonnefoy, Ahmed Raihane, Jean-Marc Chaix, Jean-Louis Gelet, Gérard Thomas

\section{To cite this version:}

Sébastien Nadler, Olivier Bonnefoy, Ahmed Raihane, Jean-Marc Chaix, Jean-Louis Gelet, et al.. Numerical Simulation Of Granular Media Under Horizontal Vibrations.. 6th International Conference on Micromechanics of Granular Media, Jul 2009, Golden, Colorado, United States. pp.725-728. hal00411051

\section{HAL Id: hal-00411051 \\ https://hal.science/hal-00411051}

Submitted on 25 Aug 2009

HAL is a multi-disciplinary open access archive for the deposit and dissemination of scientific research documents, whether they are published or not. The documents may come from teaching and research institutions in France or abroad, or from public or private research centers.
L'archive ouverte pluridisciplinaire HAL, est destinée au dépôt et à la diffusion de documents scientifiques de niveau recherche, publiés ou non, émanant des établissements d'enseignement et de recherche français ou étrangers, des laboratoires publics ou privés. 


\title{
Numerical Simulation Of Granular Media Under Horizontal Vibrations.
}

\author{
Sébastien Nadler*, Olivier Bonnefoy*, Ahmed Raihane*, Jean-Marc Chaix ${ }^{\dagger}$, \\ Jean-Louis Gelet** and Gérard Thomas* \\ ${ }^{*}$ Centre SPIN-LPMG UMR 5148, ENSMSE, 158 Cours Fauriel 42023 Cedex, France \\ ${ }^{\dagger}$ Centre SIMAP, Grenoble INP-CNRS, 1130 rue de la Piscine, BP75-Domaine Universitaire F-38402 \\ Saint-Martin d'Heres, France \\ **Ferraz-Shawmut, 6 rue de Vaucanson, 69720 Saint-Bonnet de Mure, France
}

\begin{abstract}
Sinusoidal horizontal vibrations have been applied to a parallelepiped containing rounded sand grains. To model the granular medium behavior, a commercial software based on Molecular Dynamics has been used. The influence on the rheologic behavior of many process parameters, such as the dimensionless acceleration and the frequency has been studied. The velocity, density, pressure and mass flow fields have been computed, both in dynamic and static modes and compared with experimental results. The correlation between these different parameters has been also examined.
\end{abstract}

Keywords: Granular media, vibration, shaking, horizontal, rheology, DEM, numerical simulations, modeling, stress tensor, packing fraction, densification, pressure

PACS: $45.70 . \mathrm{Cc} ; 42.70 . \mathrm{Vn}$

\section{INTRODUCTION}

The densification by vibration is a quite common process in industry: e.g. in civil engineering, a vibrating roll can be used to stabilize soils. In this work, we shall focus on another kind of application: fuses for the electric industry. They are constituted of silver blades in a ceramic box (cube, cylinder...), containing a sand packing that needs to be as compact and homogeneous as possible. Many experimental studies have been already done under vertical vibrations $[1,2]$, a few are related to horizontal vibrations [3]. Numerical studies such as in [4] have developed a hybrid hard sphere model and particle dynamics scheme to describe the influence of horizontal vibrations on the rheology of $2 \mathrm{D}$ granular media. In our laboratory, vertical and horizontal vibrations have been already studied, by Emilie Rouèche [5] and Ahmed Raihane respectively ([6] and [7]).

Hereafter, we shall focus on understanding the effect of horizontal vibrations on the packing density and on the granular motion. A numerical approach using a soft sphere DEM commercial software (PFC3D from ITASCA) allows to model some properties of the vibrated granular media. The results of numerical simulations will be compared with that of real-world experiments.

\section{EXPERIMENTAL SET UP AND OBSERVATIONS}

The sand packing is placed in a rectangular box (Figure 1). A sinusoidal displacement is then imposed to the system $\mathrm{x}=\mathrm{A} \sin \omega \mathrm{t}$. Let us call $\Gamma=\frac{\mathrm{A} \omega^{2}}{\mathrm{~g}}=\frac{\mathrm{a}}{\mathrm{g}}$ the dimensionless acceleration, $\mathrm{g}$ the gravity field $\left(g \approx 9.8 \mathrm{~ms}^{-2}\right)$, and $\mathrm{f}=\frac{2 \pi}{\omega}$ the frequency of the applied vibration. Five points of view of the transparent box made of PMMA can be chosen: North, South, East, West and upside. The internal dimensions of the box are denoted Lx, Ly, and $\mathrm{H}_{\text {sand }}$ is the height of the sand bed (Table 1). The typical volume size distribution (in diameters) of the grains used in our laboratory can be roughly approximated by a gaussian curve with a mean value of $450 \mu \mathrm{m}$ and a standard deviation of $100 \mu \mathrm{m}$. The grain shape appears to be quite spherical.

Above a critical acceleration, the rheologic behavior of the granular medium can be mainly described by two contra-rotating convection rolls in the upper region as described on Figure 2. This observation can be interpreted as follows: the vibrations create an intermittent gap along the North or South walls and the granular medium. By gravity, the grains can fall down into this gap. Then, they penetrate into the bulk, pushed horizontally and upwards by the vibration or inertial forces. Finally, when they arrive on the top of the pile, the grains may fall down again and the loop is closed [7]. Figure 3 shows the experimental velocity field. 


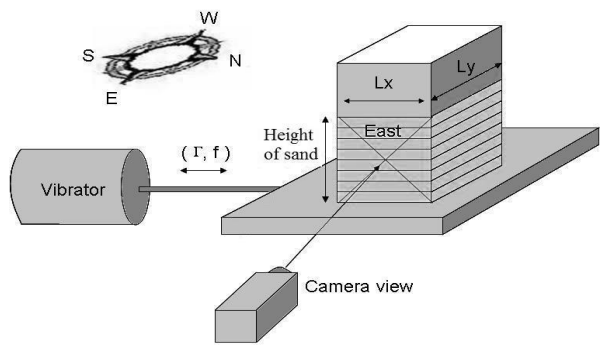

FIGURE 1. Schematic experimental setup

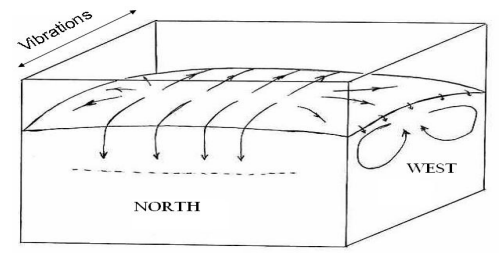

FIGURE 2. Contra-rotating convection rolls observed experimentally when $\mathrm{f}=50 \mathrm{~Hz}$ and $\Gamma>1.5$.

\section{MODELING: PROTOCOL}

Results of modeling works found in literature are quite difficult to reproduce because the set of parameters is not extensively indicated. Anyway, differences between experimental and model parameters are imposed to keep the calculation time reasonable (Table 1). Our method consists in transforming the lagrangian description natively used by the DEM software into an eulerian one and compare it with experimental results.

Lagrangian description. The modeling is based on a series of operations applied to a given assembly of spheres presenting a uniform numeric particle size distribution (PSD), randomly placed in a parallelepiped, and packed under gravity. The PSD has been chosen in order to prevent the packing from crystallization. Sinusoidal

TABLE 1. Typical parameters of the model.

\begin{tabular}{llll}
\hline Parameter & Real value & Model value & Comment, Model/Real \\
\hline Mean diameter $[\mathrm{mm}]$ & $\approx 0.45$ & 0.90 & Bigger sphere* \\
Rigidity $\left[\mathrm{N} \cdot \mathrm{m}^{-\frac{3}{2}}\right.$ ] & $2 \cdot 10^{8}$ & $2 \cdot 10^{5}$ & Smaller rigidity* \\
Density $\left[\mathrm{kg} \cdot \mathrm{m}^{-3}\right]$ & 2700 & 2700 & \\
Shear coefficient [Pascals] & $4.0 \cdot 10^{10}$ & $4.0 \cdot 10^{7}$ & $\propto$ Young modulus* \\
Poisson coefficient [] & 0.25 & 0.25 & \\
Friction (wall/ball) [] & 0.3 & 0.3 & Powder rheometer value \\
Friction (ball/ball) [] & 0.7 & 0.7 & Powder rheometer value \\
Lx [mm] & 40 & 40 & \\
Ly [mm] & 80 & $\infty$ & Model : Ly=10 periodic $\Rightarrow L y \approx \infty *$ \\
$H_{\text {Sand }}[\mathrm{mm}]$ & 55 & 55 & \\
& & & $*$ Value of the model chosen \\
& & & to minimize the calculation time \\
\hline
\end{tabular}

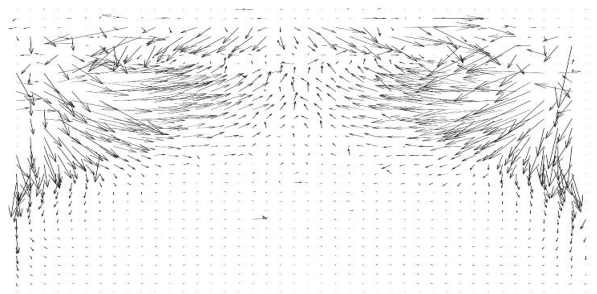

FIGURE 3. East side view, velocity field measured experimentally by Particle Image Velocimetry technics. Observation of contra-rotating convection rolls for $\Gamma=4.2$ and $\mathrm{f}=50 \mathrm{~Hz}$. Typical velocity range: $0-30 \mathrm{~mm} \mathrm{~s}^{-1}$

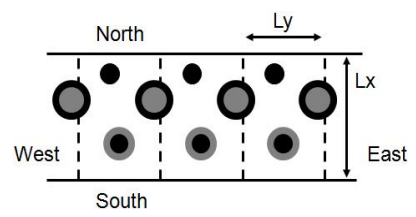

FIGURE 4. Schematic description of a periodic box

horizontal vibrations are imposed to the system. The position and velocity of each sphere are calculated step by step using the Newton's law of motion. The collisions and contacts are analyzed through a set of parameters corresponding to sand characteristics (Table 1). The deviations with the sand grains characteristics used in realworld experiments are motivated by our effort for keeping the calculation time reasonable. The position, velocity, and stress tensor associated to each sphere are computed by software at any given time. The velocity of a sphere is averaged over one period of vibration. To further reduce the calculation time, we diminished the number of spheres by using periodic boundary conditions, the unit cell size being denoted Ly (Figure 4). The influence of the periodic cell size Ly on the maximal grains velocity on North side has been analyzed. Simulations have shown that, the finite size effect vanishes when Ly exceeds approximately 10 grain diameters. For bigger values, the maximal velocity becomes independent of the unit cell size.

Eulerian description. We have created a grid related to the box, with cases indexed by a set $(\mathrm{i}, \mathrm{j}, \mathrm{k} \in N)$ of coordinates corresponding to a space position. The lagrangian representation of the software (related to the spheres) can be transformed in a eulerian representation by averaging the velocities on each case of the grid. To avoid the fluctuations due to the granular nature of the medium, the data (pressure, velocity, density) have been averaged on a series of periods. The pressure is calculated by taking the third of the trace of the stress tensor given by our software ( $\approx$ isostatic pressure). 


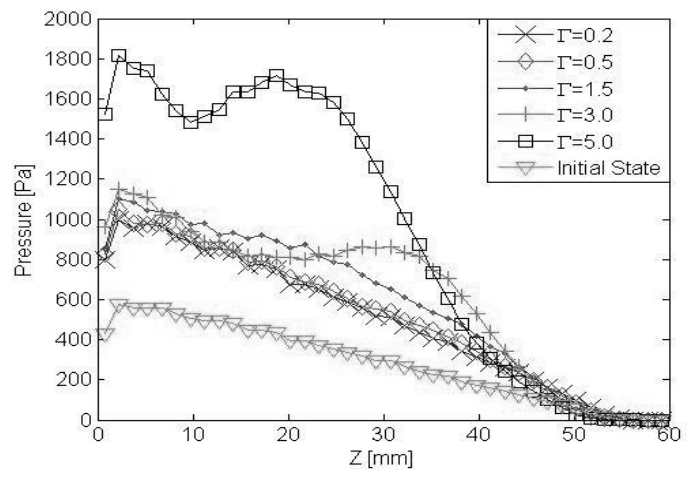

FIGURE 5. Variations of pressure profiles with the altitude $\mathrm{z}$ for initial packing and packings under vibration at $\mathrm{f}=50 \mathrm{~Hz}$. The dynamic pressure data are recorded after 100 periods.

\section{MODELING: RESULTS}

Initial State. To understand the influence of the acceleration $\Gamma$ and of the frequency $f$ on the behavior of the granular medium, an assembly of 32000 spheres is considered (Table 1). The initial state can be characterized by the vertical pressure and density profiles (Figures 5 and 9). The pressure evolution is quite linear, and reaches a maximum of $580 \mathrm{~Pa}$ for a $55 \mathrm{~mm}$ depth. This value is not far from the theoretical approximation of hydrostatic pressure for thin bed packing: $\mathrm{P} \approx \mathrm{C} \rho \mathrm{gh} \Rightarrow \mathrm{P} \approx 810 \mathrm{~Pa}$.

Transient period. When vibration are applied to the recipient, a transient period is observed. A view of the mass flow field (density_field $\times$ velocity_field) can be determined by simulation, as shown on Figure 6. The data are averaged over the ten first periods of vibrations. Two phenomena are evidenced: a compaction process, in the top of the box, corresponding to flow vectors oriented downwards, and the initiation of convection rolls. One can ask when the compaction process stops? If we calculate the $\mathrm{z}$ projection of the mean mass flow in the whole granular media $\mathbf{J}$, this variable must reach zero after a time long enough and the system must attain an equilibrium density state (the hypothesis of pseudo-stationarity of the system has been experimentally confirmed). To study this assumption, we have plotted the mean mass flow versus time for different accelerations $\Gamma$ (Figure 7). At the beginning, the mean mass flow is important and downwards oriented (compaction). Afterward, a stationary density state is attained, and the mean flow oscillates around 0 (Figure 8). The numerical results for $\Gamma=3$ and $\mathrm{f}=50 \mathrm{~Hz}$, shows that the steady states behavior began after 6 seconds of vibration (300 periods). It agrees with experimental observations.

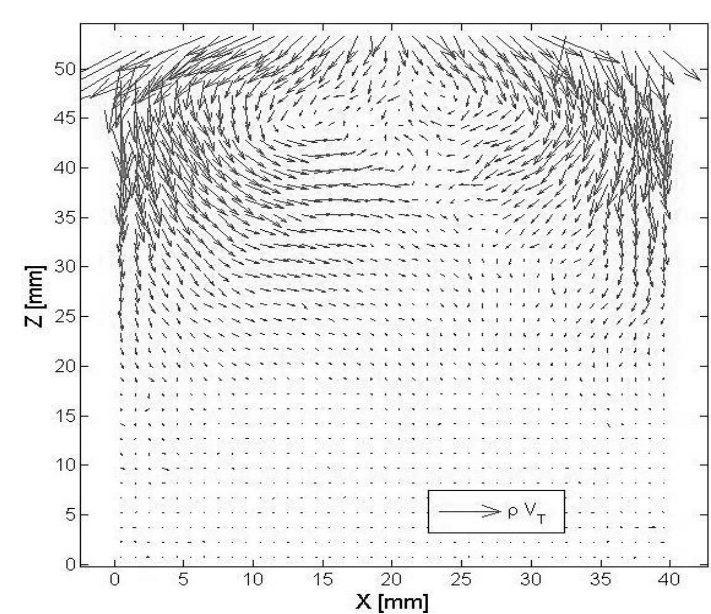

FIGURE 6. Mean flow mass field on the ten first cycles for $\Gamma=1.5, \mathrm{f}=50 \mathrm{~Hz}$ : East side view. At each location, the vector $\rho V_{T}$ is associated to a pixel. Two vortices appear in the upper part of the box while a densification process occurs.

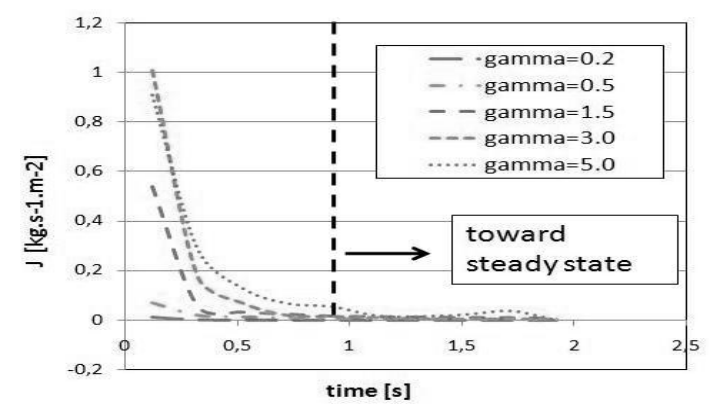

FIGURE 7. Evolution with $\Gamma$ of the downwards mean mass flow $\mathrm{J}$ vs time for $\mathrm{f}=50 \mathrm{~Hz}$. The mass flow is averaged over all representative element volume.

Steady state period. Figure 9 shows the influence of the acceleration $\Gamma$ on the density of the granular media during vibrations. We can exhibit two characteristics behaviors of the system. For a low acceleration $(\Gamma<1.5)$, the density of the bottom of the box remains equal to the density at the initial state. One can explain this phe-

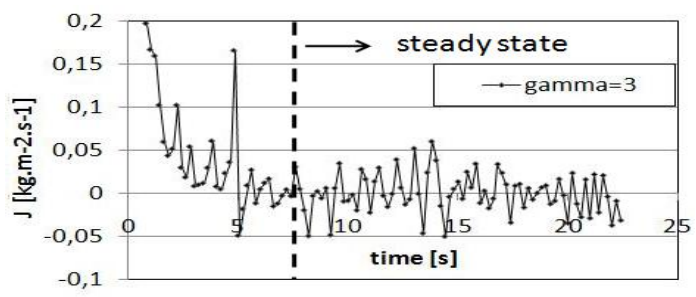

FIGURE 8. Evolution of the downwards mean mass flow $\mathrm{J}$ vs time for $\mathrm{f}=50 \mathrm{~Hz}: \Gamma=3$. The steady state behavior begins after 6 seconds. 


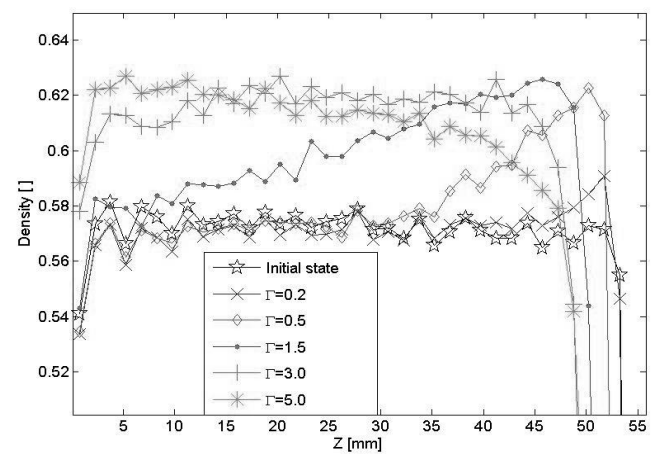

FIGURE 9. Computed evolution of the density vs. height for $\Gamma$ between 0 and $5 ; \mathrm{f}=50 \mathrm{~Hz}$, recorded after 100 periods

nomenon by looking at the pressure profile (Figure 5). The deeper are the grains in the packing, the greater is the pressure, even during the oscillations. So the grains are more constrained in the bottom layers and they need to acquire a lot of energy to change their positions. Therefore, at low accelerations, in the deeper layers, the grains are not able to move in an lower energy state; the system is blocked and the density does not change (Figure 9). For high accelerations $(\Gamma>3)$, the energy brought to the system overpasses the jamming effect due to the pressure. Therefore, the grains can move even in the deeper layers and the system get denser at the bottom of the box. However, the energy brought to the surface is so hight compared to the pressure that the granular medium becomes fluid. As a consequence, the surface density decreases.

The figure 10 shows the influence of the frequency on the density of the packing during vibration. We can observe that for an optimal range of frequency $(f=50 \mathrm{~Hz}$, $100 \mathrm{~Hz}$ ), the density is maximal, otherwise the density decreases. Notice the density drop in the upper layers for $\mathrm{f}=20 \mathrm{~Hz}$. This phenomenon is related to a sloshing flow in the fluidized upper region. The period of oscillations is large enough to allow the establishment of waves on the surface. For high frequencies, one can explain the drop of density by the small amplitude imposed in the movement. The smaller gap generated by the vibration on the North and South sides gives less opportunity for the grains to fall down and the convection process is partially or totally jammed. The possibility of rearrangement of the system becomes also less important and the global density attained can be reduced.

Relaxed period. Experimentally and numerically, we can observe a gain of density when the vibrations are stopped. The density increases from $0 \%$ in the blocked layers to $3 \%$ for the fluidized top layers of the granular packing.

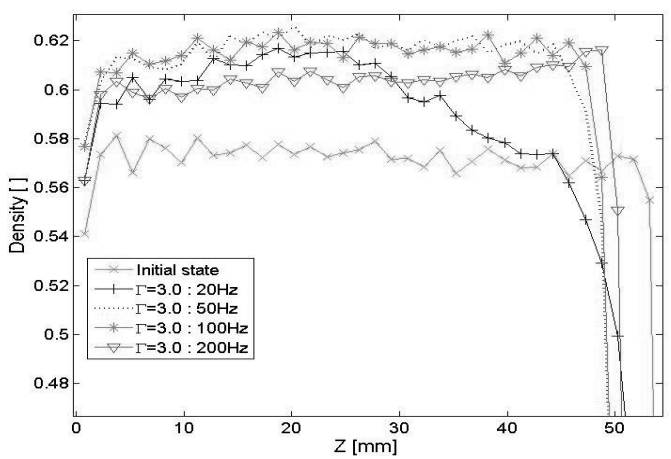

FIGURE 10. Computed evolution of the density vs. height for frequencies between 20 and $200 \mathrm{~Hz}$, recorded after 100 periods.

\section{CONCLUSION}

This numerical study enables to understand more precisely phenomena occurring during the compaction by vibration. Indeed, some data as the pressure or the velocity field in the center of the packing are only numerically accessible. Despite the choice of not very realistic parameters (sphere rigidity...) to keep reasonable computation time, the results of the modeling are surprisingly close to the ones observed in experiments (see also [6]). We have shown the influence on the system behavior of the frequency for a constant acceleration and of the acceleration for a constant frequency. The vibration amplitude was imposed to fit with these data. In order to complete our results, we shall bring to light the effect due to the vibration amplitude. We also plan to study the possible scaling up law connecting the size of the grains and their behavior.

\section{REFERENCES}

1. P. Philippe, and D. Bideau, Physical Review Letters 91, id. 104302 (2003).

2. P. Ribière, P. Richard, D. Bideau, and R. Delannay, European Physics Journal E 16(4), 415-420 (2005).

3. H. M. J. Millica Medved, Damien Dawson, and S. R. Nagel, Chaos 9 (3), 691-696 (1999).

4. P. C. Kurt Liffman, Guy Metcalfe, Physical Review Letters 79, 23 (1997).

5. E. Rouèche, G. Thomas, J.-L. Gelet, and J. Missiaen, Powder\&Grains 2, 1173-1176 (2005).

6. A. Raihane, O. Bonnefoy, J.-L. Gelet, J.-M. Chaix, and G. Thomas, "Densification of a 3D granular bed by horizontal vibrations," in Proceedings of the $\mathrm{XV}^{\text {th }}$ International Congress on Rheology (ICR2008), pp. 932-935, 2008.

7. A. Raihane, O. Bonnefoy, J.-L. Gelet, J.-M. Chaix, and G. Thomas, Powder Technology 190 (1-2), 252-257 (2008). 